\title{
The r-process nucleosynthesis: a field with still many nuclear physics questions
}

\author{
S. Goriely ${ }^{* \dagger}$ \\ Institut d'Astronomie et d'Astrophysique, CP-226, Université Libre de Bruxelles, 1050 Brussels, \\ Belgium \\ E-mail: sgorielyeastro.ulb.ac.be
}

\begin{abstract}
About half of the nuclei heavier than iron observed in nature are produced by the so-called rapid neutron capture process, or r-process, of nucleosynthesis. The identification of the astrophysics site and the specific conditions in which the r-process takes place remains, however, one of the still-unsolved mysteries of modern astrophysics. Another underlying difficulty associated with our understanding of the r-process concerns the uncertainties in the predictions of nuclear properties for the few thousands exotic neutron-rich nuclei involved, for which essentially no experimental data exist.

The present paper emphasizes some important future challenges faced by nuclear physics in this problem, particularly in the determination of the nuclear structure properties of exotic neutronrich nuclei as well as their fission properties. These quantities are particularly relevant to determine the composition of the matter resulting from the r-process. The nuclear physics difficulties are critically reviewed with special attention paid to the r-process taking place during the decompression of neutron star matter following the merging of two neutron stars.
\end{abstract}

XIII Nuclei in the Cosmos,

7-11 July, 2014

Debrecen, Hungary

${ }^{*}$ Speaker.

${ }^{\dagger} \mathrm{SG}$ is FNRS Reasearch associate 


\section{Introduction}

The r-process of stellar nucleosynthesis is invoked to explain the production of the stable (and some long-lived radioactive) neutron-rich nuclides that are heavier than iron and observed in stars of various metallicities, as well as in the solar system (for a review, see [1]). In recent years nuclear astrophysicists have developed more and more sophisticated r-process models, trying to explain the solar system composition in a satisfactory way by adding new astrophysical or nuclear physics ingredients. However, the site(s) of the r-process has (have) not been identified yet and for this reason, the r-process nucleosynthesis remains one of the main puzzles of modern astrophysics.

Progress in the modelling of type-II supernovae and $\gamma$-ray bursts has raised a lot of excitement about the so-called neutrino-driven wind environment [1-3]. However, until now a successful r-process has not been obtained ab initio without tuning the relevant parameters (neutron excess, entropy, expansion timescale) in a way that is not supported by the most sophisticated existing models. Early in the development of the theory of nucleosynthesis, an alternative to the r-process in high-temperature supernova environments was proposed. It concerns the decompression of cold neutronized matter [4]. Recently, special attention has been paid to NS mergers following the confirmation by hydrodynamic simulations that a non-negligible amount of matter, typically about $10^{-3}$ to $10^{-2} M_{\odot}$, can be ejected [5-9]. In contrast to the supernova site, investigations with growing sophistication have confirmed NS merger ejecta as viable sites for strong r-processing [1, 7 10, 12 - 14]. In particular, recent nucleosynthesis calculations [9] show that the combined contribution of both the dynamical (prompt) ejecta expelled during the binary NS-NS or NS-BH merger, as well as the neutrino and viscously driven outflows generated during the post-merger remnant evolution of the relic BH-torus systems lead to the production of r-process elements from $A \gtrsim 90$ up to thorium and uranium with an abundance distribution that reproduce extremely well the solar distribution, as well as the elemental distribution observed in low-metallicity stars $[15,16]$.

The ejected mass of r-process material, combined with the predicted astrophysical event rate (around $10^{-5} \mathrm{yr}^{-1}$ in the Milky Way [17]) can account for the majority of r-material in our Galaxy [7, 8]. In addition, the the galactic and cosmic chemical evolution of $r$ process elements have recently been studied in different evolutionary contexts [18 - 23] and, although they do not converge towards one unique quantitative picture, they all get to the conclusion that double compact star mergers may be the major production sites of r-process elements.

$\mathrm{R}$-process nucleosynthesis calculations require a reaction network including about 5000 species from protons up to $\mathrm{Z} \gtrsim 110$ lying between the valley of $\beta$-stability and the neutron drip line. All charged-particle fusion reactions on light and medium-mass elements that play a role when the nuclear statistical equilibrium freezes out need to be included in addition to radiative neutron captures and photodisintegrations. On top of these reactions, $\beta$-decays as well as $\beta$-delayed neutron emission probabilities and $\alpha$-decay rates need to be taken into account, but also fission processes, including neutron-induced, spontaneous, $\beta$-delayed and photofission, together with the corresponding fission fragment distribution (FFD) for all fissioning nucleus. All rates are based on experimental whenever available, but since only a extremely small amount of data are known experimentally, theoretical models are fundamental in providing the various predictions.

In the present contribution, we describe recent efforts to improve the predictions of nuclear masses and fission properties. Details about the r-process nucleosynthesis and the impact of the 
nuclear physics models on the abundance predictions can be found in Refs. [7-11].

\section{Nuclear mass models}

With a view to their astrophysical application in neutron-rich environments, a series of 29 nuclear-mass models have been developed for the last decade within the Hartree-Fock-Bogoliubov (HFB) approach with Skyrme and contact-pairing forces, together with phenomenological Wigner terms and correction terms for the spurious collective energy within the cranking approximation [24] (and references therein); all the model parameters have been fitted to essentially all the experimental mass data. While the first HFB-1 mass model aimed at proving that is was possible to reach a low root-mean-square (rms) deviation with respect to all experimental masses available at that time, most of the subsequent models were developed to further explore the parameter space widely or to take into account additional constraints. These include in particular a sensitivity study of the mass model accuracy and extrapolation to major changes in the description of the pairing interaction, the spin-orbit coupling or the nuclear matter properties, such as the effective mass, the symmetry energy and the stability of the equation of state.The effective interactions correspond either to a standard Skyrme force or to one of its generalized forms including the $t_{4}$ and $t_{5}$ terms which are density-dependent generalizations of the $t_{1}$ and $t_{2}$ terms, respectively. With such new terms it was possible to reproduce stiff equations of state in infinite neutron matter, as predicted by realistic calculations, and to stop the unphysical transition of neutron matter to a ferromagnetic state at all neutron-proton asymmetries.

With respect to the 2353 measured masses [25], the 29 HFB mass models give an rms deviation ranging between $0.51 \mathrm{MeV}$ for HFB-27 and $0.79 \mathrm{MeV}$ for HFB-1, as illustrated in Fig. 1 (left panel). These rms deviations can be compared to those obtained with other global mass model, such as the Gogny-HFB mass model with the D1M interaction [26] characterised by an rms of $0.79 \mathrm{MeV}$ or the finite-range droplet model (FRDM) [27] with $0.65 \mathrm{MeV}$. However, when dealing with exotic nuclei far away from stability, deviations between the HFB mass predictions can become significant, not only in the rigidity of the mass parabola, but also in the description of the shell gaps or pairing correlations [28]. The $1 \sigma$ variance between the 29 HFB mass predictions are illustrated in Fig. 1 (right panel) where deviations larger than $3 \mathrm{MeV}$ can be found at the neutron drip lines. Such large uncertainties can be interpreted as the model uncertainties (due to model defects) and are considered to be independent of parameter uncertainties, but rather a property of the given HFB model. These model uncertainties have been shown to be significantly larger than the uncertainties associated with local variations of the model parameters in the vicinity of an HFB minimum [28], as estimated using a variant of the Backward-Forward Monte Carlo method [29] to propagate the uncertainties on the masses of exotic nuclei far away from the experimentally known regions.

When considering mass models obtained in a relatively different framework, e.g the GognyHFB mass model based on the D1M interaction [26], even larger deviations are found in the mass predictions away from the experimentally known region. While deviations on the masses can reach values larger than typically $5 \mathrm{MeV}$ for exotic neutron-rich nuclei [26], neutron capture rates may deviate by 3 to 5 orders of magnitude with respect to the predictions obtained with the HFB masses. Such deviations by far exceeds what is acceptable for nucleosynthesis applications. For this reason, 

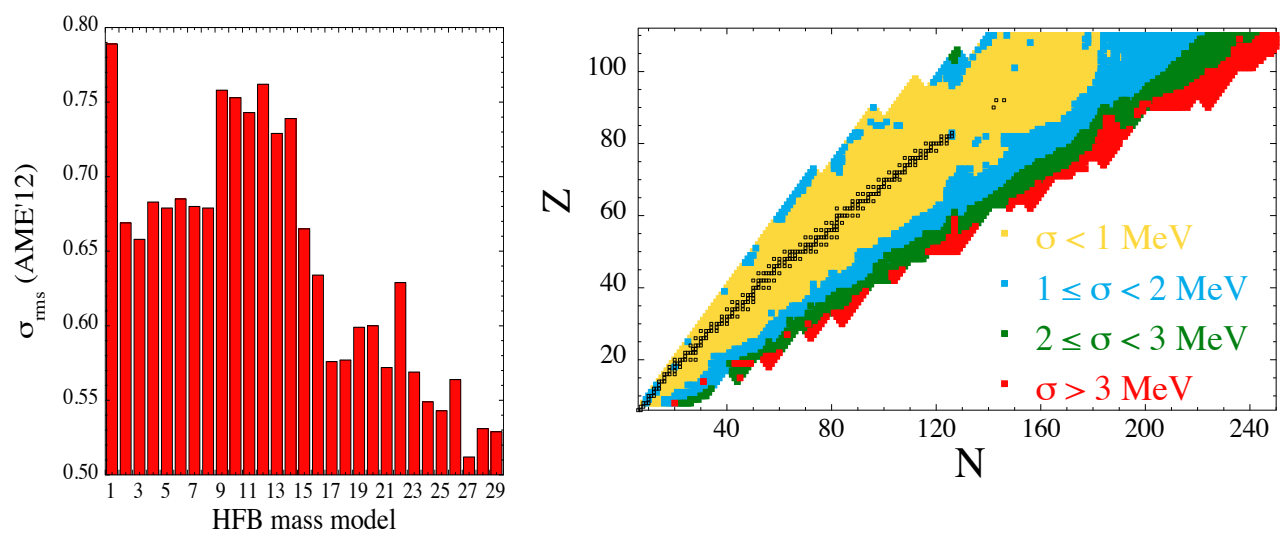

Figure 1: Left panel: Representation of the rms deviation (in MeV) for the 29 Skyrme-HFB mass models, labeled from HFB-1 to HFB-29. The rms deviation is calculated with respect to the 2353 measured masses [25]. Right panel: $1 \sigma$ uncertainty corresponding to the 29 Skyrme-HFB mass models for all the 8500 nuclei included in the mass tables from $\mathrm{Z}=8$ up to $\mathrm{Z}=110$.

further improvements of the mass model is needed. These include development of relativistic as well as non-relativistic mean field models, but also the inclusion within such approaches of the state-of-the-art corrections, like the quadrupole or octupole correlations by the Generator Coordinate Method and a proper treatment of odd- $A$ and odd-odd nuclei with time-reversal symmetry breaking. Such models should reproduce not only nuclear masses at best, but also as many experimental observables as possible. These include charge radii and neutron skin thicknesses, fission barriers and shape isomers, spectroscopic data such as the $2^{+}$energies, moments of inertia, but also infinite (neutron and symmetric) nuclear matter properties obtained from realistic ab-initio calculations as well as specific observed or empirical properties of neutron stars, like their maximum mass or mass-radius relations [30].

\section{Fission properties}

Detailed fission paths have been recently determined on the basis of the Skyrme-HFB model [31] which has proven its capacity to estimate the static fission barrier heights with a relatively high degree of accuracy. The HFB model corresponds to a standard mean-field calculation based on an effective Skyrme interaction. Of particular interest for fission application, the calculation includes all the quadrupole, octupole and hexadecapole deformation degrees of freedom, as well as a semi-microscopic quadrupole correlation energy based on the cranking model which turns out to be crucial for a proper description of the nuclear surface at large deformations.

The primary (i.e. the highest) HFB-14 barriers are compared with the empirical ones [32] in Fig. 2 where differences up to $1 \mathrm{MeV}$ can be observed. As shown in Fig. 2, similar types of deviations are found with the other global models available. These concern the macroscopicmicroscopic approach with the Finite-Range Liquid Drop Model (FRLDM) [33], the ExtendedThomas-Fermi plus Strutinsky Integral (ETFSI) model [34], the Thomas-Fermi model [35] (MS99) 


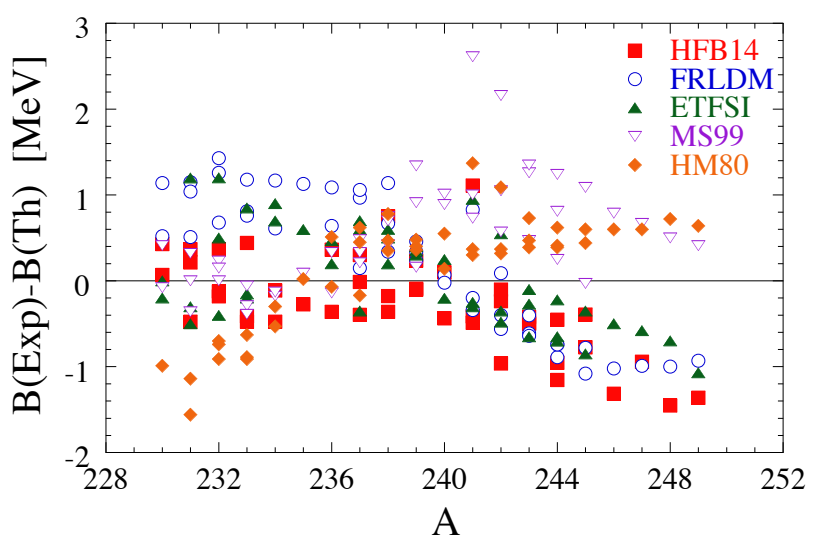

Figure 2: (Color online) Deviations between 45 empirical primary fission barriers [32] and those predicted by the HFB-14 [31], FRLDM [33], ETFSI [34], MS99 [35] and HM80 [36] global models for $88 \leq Z \leq 96$.

as well as the droplet model of Howard and Möller [36] (HM80). With respect to the 45 empirical primary fission barriers shown in Fig. 2, the rms deviation amounts to $0.60 \mathrm{MeV}$ for HFB-14, 0.81 MeV for FRLDM, 0.57 MeV for ETFSI, $0.82 \mathrm{MeV}$ for MS99 and 0.66 MeV for HM80. At this stage, no theoretical models can claim to provide predictions of barrier heights with a global accuracy better than $0.5-1 \mathrm{MeV}$ (in the best case). This comparison clearly shows that there is still a lot of room for improvement of global models in the prediction of fission barriers. Clearly, when dealing with exotic neutron-rich nuclei, much larger uncertainties affect the predictions [11].

Based on the HFB-14 fission paths, the transmission through the fission barrier has been estimated and used to derive the spontaneous, $\beta$-delayed, neutron-induced and photo fission rates $[11,37]$. The main fission regions by one of these four fission processes are illustrated in Fig. 3 (left panel). For comparison, a similar calculation is made based on the MS99 fission barriers [35]. In this case study, all the HFB-14 fission paths are renormalized, so that for each nucleus the highest barrier corresponds now to the highest MS99 barrier. The latter usually predicts lower barriers than the mean-field approach [11], so that many more nuclei are found to be affected by fission processes when use is made of the MS99 barriers, as shown in Fig. 3 (right panel).

\section{Fission fragment distribution}

The FFD as well as the number of emitted neutrons play a key role in nucleosynthesis simulations since it defines the light species that will be produced by the fission recycling [10,11]. Both the $Z$ - and $A$-dependencies of the fragment distribution need to be determined for all potentially fissioning nucleus. Since the widely-used Gaussian model of Kodoma \& Takahashi [38], a number of new global scission-point models have recently been proposed and extended to exotic nuclei for astrophysical applications. These include in particular the the so-called SPY model, corresponding to a renewed statistical scission-point model based on microscopic ingredients [39] and the so-called GEF model estimating the properties of the fission fragments and the emitted neutrons and photons in a global and semi-empirical way [41]. 

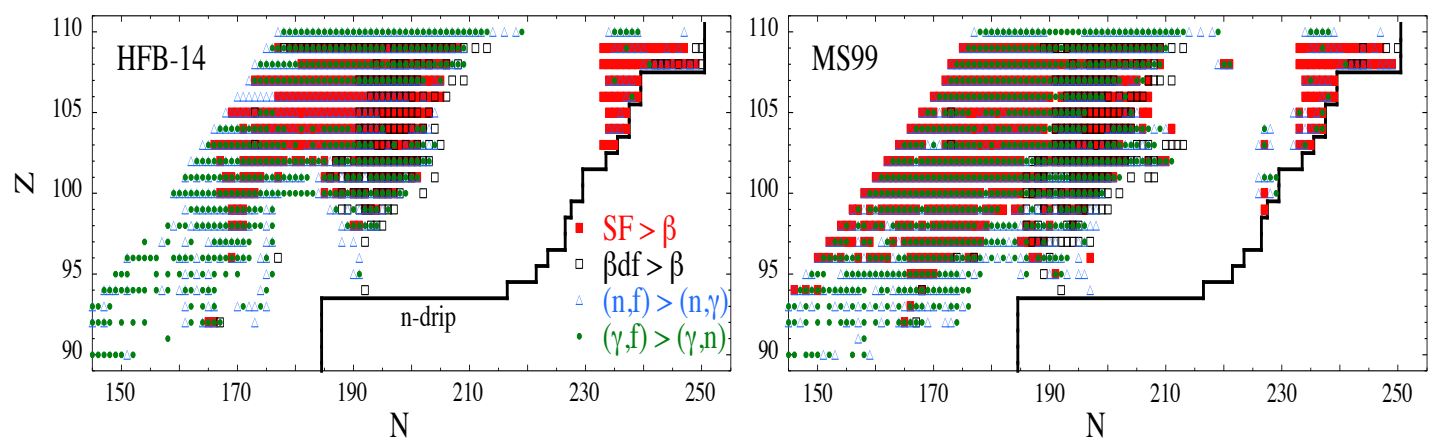

Figure 3: Representation of dominant fission regions in the $(N, Z)$ plane. Nuclei for which spontaneous fission is estimated to be faster than $\beta$-decays are shown by full squares, those for which $\beta$-delayed fission is faster than $\beta$-decays by open squares, those for which neutron-induced fission is faster than radiative neutron capture at $T=10^{9} \mathrm{~K}$ by open triangles and those for which photo-fission at $T=10^{9} \mathrm{~K}$ is faster than photoneutron emission by closed circles. The left panel is obtained with the HFB-14 fission path [31], while the right panel is derived assuming the fission paths are renormalized on the MS99 primary barriers [35].

Both the SPY and GEF models predict significantly different FFDs, as illustrated in Fig. 4 where the yields of eight $A=278$ isobars are shown. The FFD of these isobars have a direct imprint on the final r-abundance distribution resulting from NS mergers [10,11]. In the GEF case, the fragment distribution are essentially symmetrical for these particular fissioning nuclei (except for $Z=97-99$ ), whereas a 4-peak distribution is predicted by SPY for all the corresponding isobars. Such doubly asymmetric fragment distributions have never been observed experimentally and can be traced back to the predicted Gogny-HFB potential energies at large deformations for the neutron-rich fragments favored by the $A \simeq 278$ fission [10]. Detailed Gogny-HFB calculations of the potential energy surface in the parent fissioning nucleus have confirmed qualitatively the presence of these two asymmetric fission modes [10, 42].

Finally, the SPY and GEF predictions of the average number of evaporated neutrons for each spontaneously fissioning nucleus is also of prime importance for the r-process nucleosynthesis [11]. This average number is seen to reach, for both models, values of about 4 for the $A \simeq 278$ isobars and maximum values of $\sim 14$ for the heaviest $Z \simeq 110$ nuclei lying at the neutron drip line. The implications for the r-process nucleosynthesis are discussed in Refs. [10, 11].

\section{Conclusions}

Decompressed NS matter remains a viable site for the r-process, which is extremely robust with respect to many astrophysical uncertainties. However, the estimated abundance distribution remains rather sensitive to the adopted nuclear models. The material ejected from the dynamical merging phase is composed almost exclusively of $A>140$ nuclei, and in particular the $A \simeq 195$ third r-process peak appears in proportions similar to those observed in the solar system, deviations resulting essentially from the still difficult task to predict neutron capture and $\beta$-decay rates, as well as the number of prompt fission neutrons emitted for exotic neutron-rich nuclei. In this respect, 


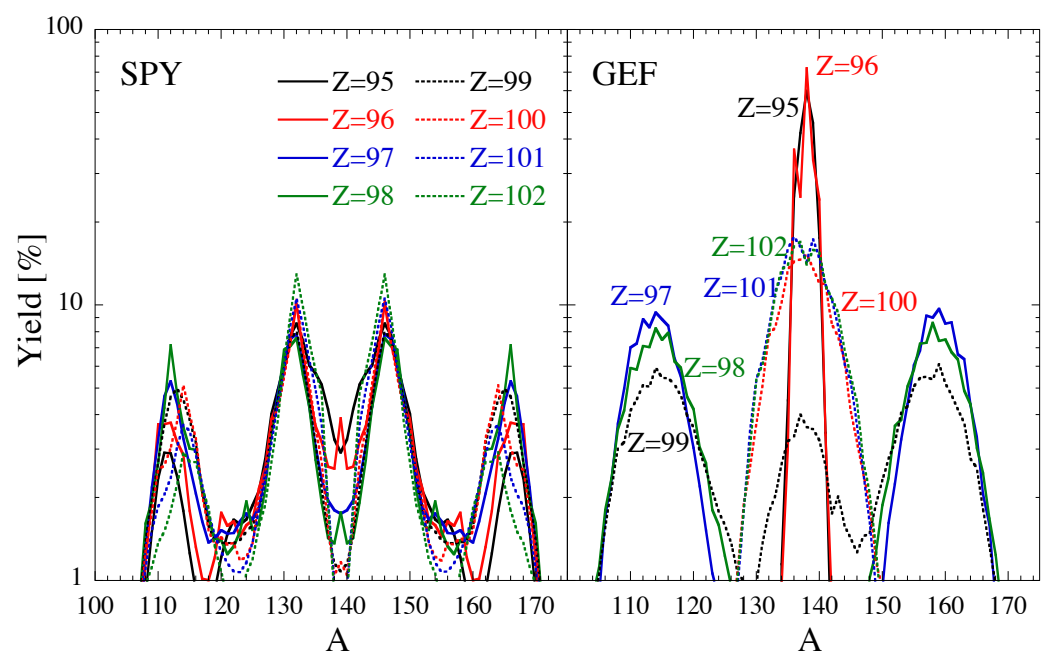

Figure 4: (Color online) FFDs predicted by the SPY (left panel) and GEF models (right panel) for $8 \mathrm{~A}=278$ isobars.

nuclear masses remain the most basic ingredient that needs to be determined more reliably far away from stability. More work in developing microscopic mass models is needed. The abundance of $110 \lesssim A \lesssim 170$ nuclei is found to depend sensitively on the fission processes affecting the isobaric chains around $A \simeq 278$ at the end of the neutron irradiation when the abundant $N=184$ nuclei decay back to the valley of stability. Both the fission probabilities and FFDs of nuclei in this region play a crucial role in shaping the production of $110 \lesssim A \lesssim 170$ nuclei. For these reason, fission properties need to be determined on the basis of sound and as microscopic as possible nuclear models. Phenomenological, empirical or systematics extrapolations in the exotic neutronrich region should be avoided because of their lack of predictive power. Mean field calculations are now available and can be used to estimate the potential energy surfaces and associated collective inertia tensors. Future systematic analysis of the collective fission dynamics through a study of the time evolution of the compound system on the basis of a time-dependent Schrödinger equation $[42,43]$ could shed light on the fission properties of exotic nuclei involved in the r-process.

\section{References}

[1] M. Arnould, S. Goriely, T. Takahashi, Phys. Rep. 450 (2007) 97

[2] H.-T. Janka, Ann. Rev. Nuc. Part. Science 62 (2012) 407

[3] Wanajo S, Janka H-T, Müller B 2011 Astrophys. J. Lett. 726 L15

[4] J.M. Lattimer, D.N. Schramm, Astrophys. J. 192 (1974) L145

[5] M. Ruffert, H.-T. Janka, G. Schäfer, Astron. Astrophys. 311 (1996) 532

[6] S. Rosswog, et al., Astron. Astrophys. 341 (1999) 499

[7] S. Goriely, A. Bauswein, H.-T. Janka Astrophys. J. Lett. 738 (2011) L32. 
[8] A. Bauswein, S. Goriely, H.-T. Janka, Astrophys. J. 773 (2013) 78.

[9] O. Just, A. Bauswein, R. Ardevol Pulpillo, S. Goriely, H.-T. Janka, arXiv:1406.2687 (2014).

[10] S. Goriely, et al., Phys. Rev. Lett. 111, (2013) 242502.

[11] S. Goriely, Eur. Phys. J. A, submitted (2014).

[12] S. Goriely, P. Demetriou, H.-T. Janka, J.M. Pearson, Nucl. Phys. A 758 (2005) 587c

[13] B.D. Metzger, et al., Mont. Not. Roy. Astr. Soc. 406 (2010) 2650 (2010).

[14] O. Korobkin, S. Rosswog, A. Arcones, C. Winteler, Month. Not. Roy. Astro. Soc. 426 (2012) 1940.

[15] C. Sneden, J.J. Cowan, R. Gallino, Ann. Rev. Astron. Astrophys. 46 (2008) 241.

[16] I.U. Roederer, et al., Astrophys. J. 724 (2010) 975.

[17] M. Dominik et al. Astrophys. J. 759 (2012) 52.

[18] F. Matteucci, et al., Month. Not. Roy. Astro. Soc. 438 (2014) 2177.

[19] Y. Komiya, S. Yamada, T. Suda, M.Y. Fujimoto, Astrophys. J. 783 (2014) 132.

[20] N. Mennekens, D. Vanbeveren, Astron. Astrophys. 564 (2014) A134.

[21] S. Shen, R. Cooke, E. Ramirez-Ruiz, P. Madau, L. Mayer, J. Guedes, arXiv:1407.3796 (2014).

[22] F. van de Voort, E. Quataert, P.F. Hopkins, D. Keres, C.-A. Faucher-Giguere, arXiv:1407.7039 (2014).

[23] E. Vangioni, S. Goriely, F. Daigne, P. Frano̧is, Astron. Astrophys., submitted (2014).

[24] S. Goriely, Nucl. Phys. A (2014) in press.

[25] G. Audi, et al., Chinese Physics C 36 (2012) 1287.

[26] S. Goriely, S. Hilaire, M. Girod, S. Péru, Phys. Rev. Lett. 102 (2009) 242501.

[27] P. Möller, J. R. Nix, W.D. Myers, and W.J. Swiatecki, At. Data Nucl. Data Tables 59 (1995) 185.

[28] S. Goriely, R. Capote, Phys. Rev. C 89 (2014) 054318.

[29] E. Bauge, and P. Dossantos-Uzarralde, J. Korean Phys. Soc. 59 (2011) 1218.

[30] A. F. Fantina, N. Chamel, J. M. Pearson, S. Goriely, Astron. Astrophys. 559 (2013) A128.

[31] S. Goriely, M. Samyn, J.M. Pearson,Phys. Rev. C 75 (2007) 064312

[32] R. Capote, et al., Nuclear Data Sheets 110 (2009) 3107.

[33] P. Möller, et al., Phys. Rev. C 79 (2009) 064304.

[34] A. Mamdouh, J.M. Pearson, M. Rayet, F. Tondeur, Nucl Phys. A 679 (2001) 337.

[35] W.D. Myers, W.J. Swiatecki, Phys. Rev. C 60 (1999) 014606.

[36] W.M Howard, P Möller, At. Nucl. Data Tables 25 (1980) 219.

[37] S. Goriely, S. Hilaire, A.J. Koning, M. Sin, R. Capote, Phys. Rev. C 79 (2009) 024612.

[38] T. Kodoma, K. Takahashi, Nucl. Phys. A 239 (1975) 489.

[39] S. Panebianco, et al., Phys. Rev. C 86, (2012) 064601.

[40] S. Hilaire, M. Girod, Eur. Phys. J. A 33 (2007) 237.

[41] K.-H. Schmidt and B. Jurado, Phys. Procedia 31 (2012) 147.

[42] N. Dubray, D. Regnier, Computer Phys. Comm. 183 (2012) 2035.

[43] H. Goutte et al., Phys. Rev. C 71 (2005) 024316. 